\title{
"Multi-beam" capabilities for high precision astrometry at low frequencies using VLBI
}

\author{
Sergio Jiménez-Monferrer* \\ Universitat de València (Spain), ICRAR (UWA, Perth, Australia) \\ E-mail: sergio.jimenez@uv.es
}

\section{María J. Rioja}

ICRAR/UWA (Perth, Australia), OAN (Spain)

E-mail: maria.rioja@uwa.edu.au

\section{Richard Dodson}

ICRAR/UWA (Perth, Australia)

E-mail: richard.dodson@uwa.edu.au

\section{Oleg Smirnov}

ASTRON (Dwingeloo, The Netherlands)

E-mail: smirnoveastron.nl

\section{J. C. Guirado}

Universitat de València (Spain)

E-mail: jose.c.guirado@uv.es

We are carrying out a simulation study to characterise the advantages of VLBI with multiple beams, which will be a feature of the next generation of instruments. We will focus on VLBI astrometric measurements at lower frequencies (1.4 GHz and below). For our simulations, we have selected a network consisting of ASKAP, the Australian SKA precursor, plus existing Australian antennas from the LBA (Long Baseline Array) and the new antenna in New Zealand (figure 1a). We have used different models to represent the ionospheric turbulences and frequencies. The preliminary results show an improvement of an order of magnitude in the astrometric precision achieved using multiple calibrators with angular separations of a few degrees around the target, with respect to a single nearby $\left(1^{\circ}\right.$ away) calibrator. Such astrometric precision is comparable to using in-beam phase referencing with a calibrator some arcminutes away. We plan to expand our simulations to include other networks with multi-beam capability, and ultimately SKA-like configurations.

10th European VLBI Network Symp. and EVN Users Meeting: VLBI and the new generation of radio arrays September 20-24, 2010

Manchester, UK

\footnotetext{
*Speaker.
} 


\section{Scientific Rationale}

The ionospheric contribution is the dominant source of errors in VLBI observations at low frequencies, and limits the application of conventional phase referencing techniques for astrometric measurements. The conventional approach consists of mapping a target source using the observations from a single calibrator, made at a different time and along a different line of sight. Because of the unpredictability of the temporal and spatial fluctuations in the plasma density, this introduces differential path variations between consecutive observations of the two sources, even using fast source switching and small separation angles.

Hence, current high precision astrometric measurements are restricted to the very few targets with a suitable in-beam calibrator ( $\sim$ few arcmins away). Alternatively, the use of multiple calibrators (at wider angular separations) has been shown to be a useful approach to compensate the ionospheric residuals ([1], [2]). In this context, the multi-beam capability of the next generation of low frequency instruments offers the prospect of a wide application of high precision astrometric measurements to many sources. Using simultaneous observations of multiple calibrators $(\sim$ few degrees away), surrounding the target source, allows for the compensation of both temporal and spatial fluctuations in the ionosphere. We refer to this as multi-view (or cluster-cluster) mode, as recently reviewed in [3]. Here we present results from a work in progress to characterise the astrometric precision enabled with multi-view observations.

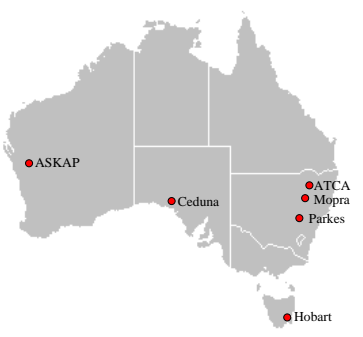

(a) Telescopes

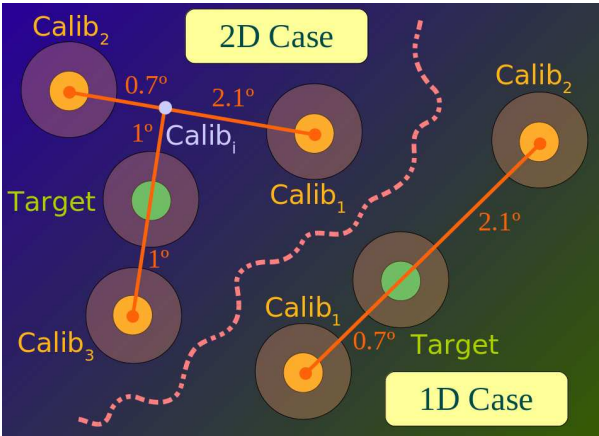

(b) Arrangements

Figure 1: (a) Telescopes used for the simulations. (b) Source distribution cases: 2 calibrators aligned with the target source (1D case), and 3 calibrators surrounding the target source (2D case). Calib ${ }_{i}$ is a virtual source obtained by combining Calibrators 1 and 2 .

\section{Simulation Studies}

We performed simulation studies to compare the astrometric precision achieved using VLBI phase referencing observations of a target source at low frequencies, using both approaches, with one calibrator and with multiple calibrators. We used CASA and MeqTrees ([4]) to generate the synthetic phase referencing data sets including various ionospheric models. We will discuss here two of these models: (i) a sinusoidal ionospheric model, with different spatial scales and (ii) a Kolmogorov model, with different observing frequencies. The simulated schedule consisted mainly of rapid switching ( $\sim 1$ minute) between sources. Some limited investigation with simultaneous 
observations of multiple sources (i.e. multi-view mode) have also been done. The design and ionospheric parameters used in the simulations are listed in Tables 1 and 2.

The data analysis was carried out following standard procedures for phase referencing analysis within AIPS for the single calibrator case; for the multi-calibrator case, we used the external program Octave for a 2-D spatial interpolation from the calibrators to the target source positions. In all cases, the phase referenced maps of the target source where generated using the task IMAGR in $A I P S$.

We measured the peak flux and astrometric offset in the phase referenced maps to asses the quality and astrometric errors in each case.

\begin{tabular}{cccccc} 
Network & Source Params. & HA Range & Distribution & Ionospheric Model & Scheduling \\
\hline ASKAP, & $0.5 \mathrm{Jy}$ & $-4 \mathrm{~h} \rightarrow 4 \mathrm{~h}$ & 1D / 2D & Sinusoidal & Rapid Switching \\
LBA \& NZ & (zero noise) & & (see fig. 1b) & \& Kolmogorov & \& Simultaneous
\end{tabular}

Table 1: Design setup used in our simulations.

\begin{tabular}{|c|c||c|c|}
\hline \multicolumn{2}{|c||}{ Sinusoidal } & \multicolumn{2}{c|}{ Kolmogorov } \\
\hline Height & $300 \mathrm{~km}$ & Height & $300 \mathrm{~km}$ \\
\hline TEC0 & $10 \mathrm{TECU}$ & TEC0 & $10 \mathrm{TECU}$ \\
\hline$\Delta$ TEC & $1 \mathrm{TECU}(10 \%)$ & $\Delta$ TEC & $1 \mathrm{TECU}(10 \%)$ \\
\hline Velocity & $0 \mathrm{~km} / \mathrm{h}$ & Velocity & $600 \mathrm{~km} / \mathrm{h}, 50 \mathrm{~km} / \mathrm{h}$ \\
\hline Spatial Scale & $50,75,100,200 \& 400 \mathrm{~km}$ & Inner / Outer Scale & $1 \mathrm{~km} / 1024 \mathrm{~km}$ \\
\hline Frequency & $1420 \mathrm{MHz}$ & Frequencies & $1420,1200,950,700 \& 327 \mathrm{MHz}$ \\
\hline
\end{tabular}

Table 2: Ionospheric models used in our simulations.

\section{Results}

Our simulations show that the astrometric precision achieved using multi-view mode, with three calibrators a few degrees away, is always superior to that using a single calibrator 1 degree away. We have confirmed that the improved multi-view astrometric precision (around $100 \mu$ as for the larger ionospheric spatial scales) corresponds to that obtained with an in-beam calibrator a few arcmin away (figure 2). Also, according to our simulations, the flux recovery in the phase referenced images of the target source is significantly higher (above 99\% for the larger spatial scales) using multiple calibrators than with a single one.

Moreover, we find that the rapid switching schedule shows significant losses in recovered flux that are reduced by using truly simultaneous multi-view mode. This is consistent with the assumption that the losses are due to the dynamic component of the ionosphere ([5]).

\section{Future work}

We plan to expand our simulation studies repeating the process for different spatial scales (sinusoidal model) and frequencies (Kolmogorov model), along with different source distributions. 

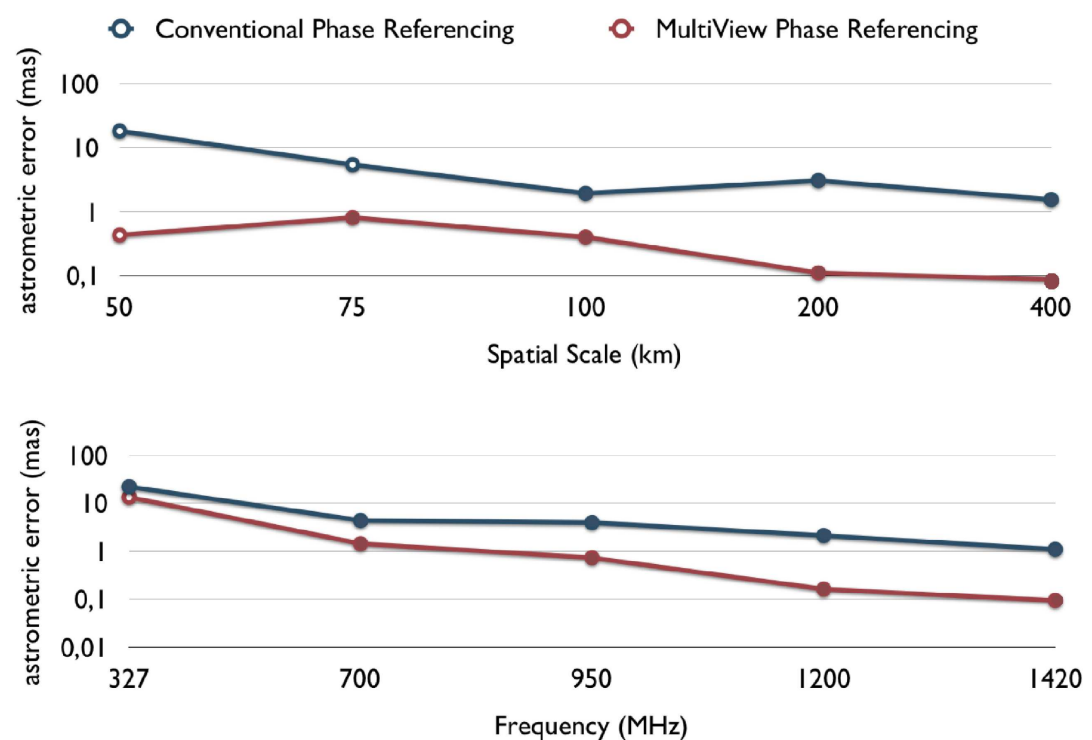

Figure 2: Astrometric errors resulting from our simulations using sinusoidal (top) and Kolmogorov (bottom) models, with a single calibrator (blue line) and multiple calibrators (red line). Filled circles represent a flux recovery larger than $60 \%$ in the phase referenced maps, meaning reliable astrometric data.

A complete simulation study will serve to explore the astrometric performance under different circumstances and to design the observing strategy on a case-by-case basis to optimise the outcome. Ultimately, we will perform new simulations including other networks with multi-beam capabilities, having SKA-like configurations as our final goal.

\section{Acknowledgments}

This work was performed during a 4-months visit to ICRAR by Sergio Jiménez-Monferrer, funded by the Spanish Ministry of Science and Innovation. This activity is partially supported through the grants AYA2006-14986-C02-02 and AYA2009-13036-C02-02.

\section{References}

[1] E. B. Fomalont and S. Kopeikin, Phase Referencing Using Several Calibrator Sources, 2002, in proceedings of the 6th European VLBI Network Symposium, 53.

[2] M. J. Rioja et al., VLBI observations in Cluster-Cluster mode at $1.6 \mathrm{GHz}, 2002$, in proceedings of the 6th European VLBI Network Symposium, 57.

[3] M. J. Rioja et al., Revisited "Cluster-Cluster" VLBI with future multi-beam low frequency radio interferometers, 2009, in proceedings of the 8th International e-VLBI Workshop, EXPReSO9, PoS(EXPReS09)014.

[4] J. E. Noordam and O. M. Smirnov, The MeqTrees software system and its use for third-generation calibration of radio interferometers, A\&A, in press.

[5] Y. Asaki et al., Verification of the Effectiveness of VSOP-2 Phase Referencing with a Newly Developed Simulation Tool, ARIS, 2007, in Publications of the Astronomical Society of Japan, PASJ 59, 397. 